DE

M E D I C I N A

T R O P I C A L

$\mathrm{DE}$

S ÃO PAULO

JOURNAL OF THE SÃO PAULO INSTITUTE OF TROPICAL MEDICINE

${ }^{1}$ Universidade Federal do Pará, Belém, Pará, Brazil

${ }^{2}$ Centro Universitário do Estado do Pará, Belém, Pará, Brazil

Correspondence to: Carla Andrea Avelar Pires

Universidade Estadual do Pará, Tv

Perebebuí, 2623, Belém, PA, Brazil

Tel: +55 91 3131-1704

E-mail: carlaavelarpires@gmail.com

Received: 26 June 2019

Accepted: 4 December 2019

\section{Kaposi's sarcoma in a HIV-positive patient: an exuberant and widespread case report in the Amazon}

\author{
Carla Andrea Avelar Pires ${ }^{[1}$, Julius Caesar Mendes Soares Monteiro', \\ Rhyan Meninea Rego', Victoria Juliana Campos Lodi ${ }^{\circledR 2}$
}

\section{ABSTRACT}

A 37-year-old male patient, Fitzpatrick skin phototype IV, a student living in Belem, Amazon region, in 2015 had a confirmed diagnosis of acquired immunodeficiency virus (HIV) infection, but did not initiate antiretroviral treatment at his own option. Three years after the diagnosis, erythematous maculae appeared on the dorsum of the nose with rapid progression to the entire face, with posterior diffuse infiltration and appearance of nodules on the chin and shoulder. In December 2018, the patient presented with exacerbation of the condition with an increase in infiltrated violaceous plaques and disseminated violaceous nodules. A histopathological biopsy of the skin was performed, confirming the diagnosis of angiomatoid proliferation suggestive of Kaposi's sarcoma (KS), with an important dissemination of this disease to the noble organs. In addition, it is important to note that he only started antiretroviral therapy (ART) after the exacerbation of Kaposi (December 2018). In such cases, chemotherapy associated with ART is crucial for the treatment and follow-up of the patient, since Kaposi's sarcoma develops relatively low in patients who do not have immunodeficiency.

KEYWORDS: Kaposi’s sarcoma. HIV. Antiretroviral therapy (ART).

\section{INTRODUCTION}

HIV infection has become a worldwide public health problem. Over 36 million people are estimated to be infected with HIV, and approximately 1.1 million deaths were attributed to this infection in 2015. In addition, by the end of 2015, over 2.1 million new cases were identified. Several skin diseases are almost unique to HIV-infected individuals, such as oral hairy leukoplakia, bacillary angiomatosis and Kaposi's sarcoma ${ }^{1}$.

Kaposi's sarcoma (KS) is an HIV-related vascular neoplasm associated with human herpes virus 8 infections. Cutaneous KS presents as red, violaceous (purple), or brown lesions, from macules, patches and papules to nodules or tumors. KS can present as skin or mucomembranous lesions, however, disseminated disease may affect any organ. The most common sites of disease dissemination include the skin, mucosal surfaces, respiratory tract and lymph nodes, and extensive disseminated disease is often associated to lymphedema².

\section{CASE REPORT}

In 2015, a 37-year-old male patient, Fitzpatrick skin phototype IV, a student living in Belem municipality, Para State, in the Amazon region, had a confirmed diagnosis 
of acquired immunodeficiency virus (HIV) infection, but he chose not to start the antiretroviral treatment at that time. Three years after the diagnosis, erythematous maculae appeared on the dorsum of the nose with rapid progression to the entire face, with posterior diffuse infiltration and appearance of nodules on the chin and shoulder (Figure 1a). In July 2018, lesions had spread throughout the body (Figure 1b, 1c and 1d) and periorbital edema was present. At this time, the patient had a CD4 $+\mathrm{T}$ lymphocyte count of 142 cells $/ \mathrm{mm}^{3}, \mathrm{~T}$ CD $8+1,593$ cells $/ \mathrm{mm}^{3}$ and viral load (CV) of 1,556,502 copies $/ \mathrm{mm}^{3}$. In view of the diagnostic hypothesis of Kaposi's sarcoma (KS) associated to AIDS, the patient accepted to initiate the antiretroviral therapy (ART) with the Tenofovir (TDF), Lamivudine (3TC) and Dolutegravir (DTG).
In December 2018, the patient presented exacerbation of symptomatology and increased infiltrated violaceous plaques as well as disseminated violaceous nodules. In view of the severity of the case and suspicion of the presence of lesions in multiple organs and due to the worsening of the clinical symptomatology, an investigation of the possible dissemination to inner organs was carried out and the patient was admitted to the infectious diseases ward of the university hospital, where biopsy of the skin was performed for histopathological exams that confirmed the diagnosis of angiomatoid proliferation suggestive of Kaposi's sarcoma (KS). The upper digestive endoscopy found a number of violaceous plaques $(5-7 \mathrm{~cm})$ in the esophagus (Figure 1e), and elevated sessile violaceous lesions $(5 \mathrm{~mm})$ in the stomach and duodenum (Figure 1f) that the diagnosis of

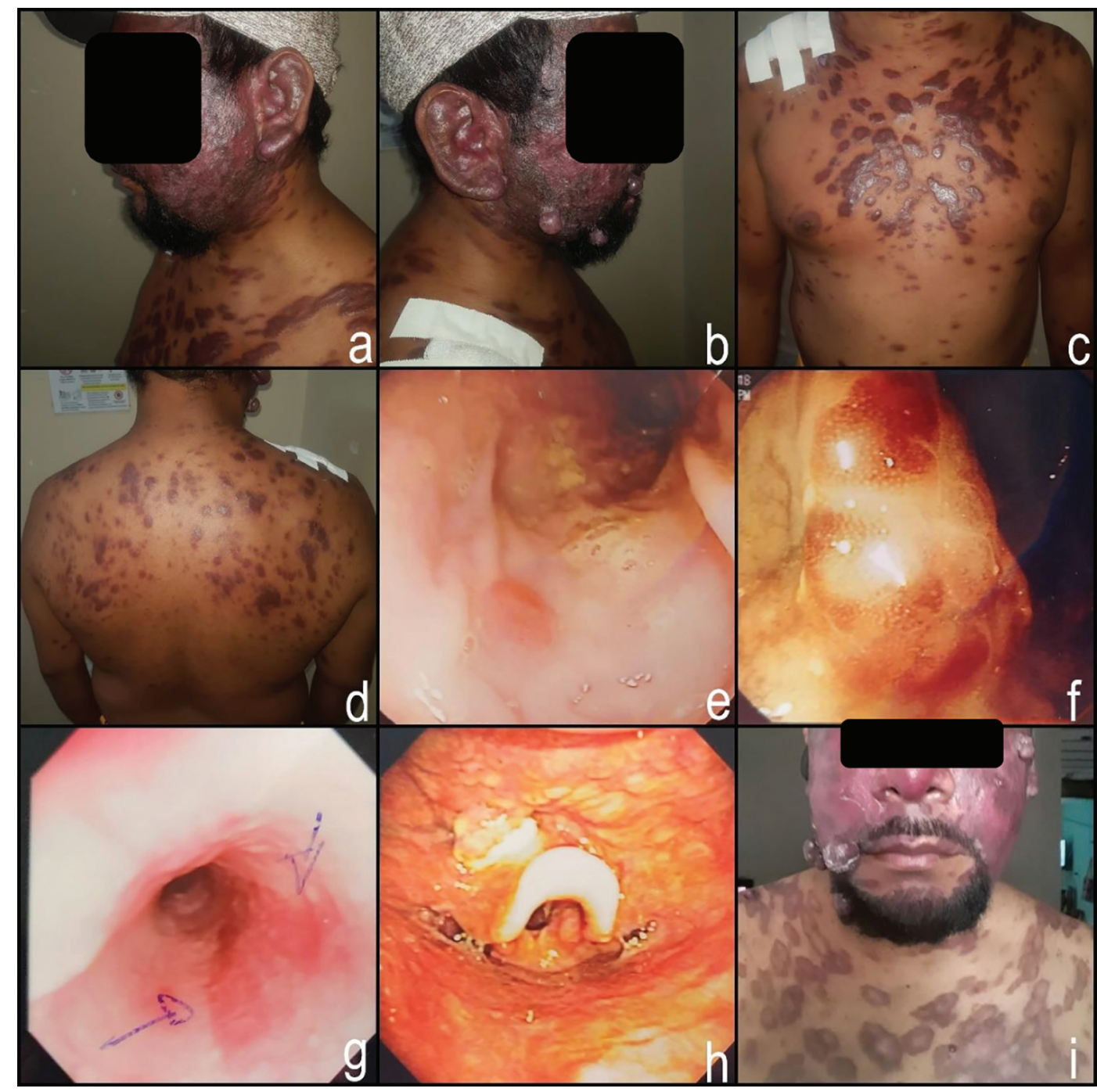

Figure 1 - Nodules and erythematous-infiltrated plaques with clear borders, irregular, confluent, coalescent and disseminated contours on the face (a), auricular pavilion (b), trunk (c) and limbs (d); (e) purple 5-7 cm plaques from the esophagus, proximal to the distal point; (f) Elevated sessile, violet, $5 \mathrm{~mm}$ lesion in the antral mucosa; (g) elevated, violaceous, hardened lesion covered with fibrin in the ileocecal valve; (h) a cyst-like lesion in the left supraglottic region; (i) aspect of lesions after chemotherapy. 
Kaposi's sarcoma lesions after microscopy. The patient underwent a colonoscopy, that evidenced a violaceous lesion $(3 \mathrm{~cm}$ ) in the cecal ileum (Figure 1g) and others lesions $(5 \mathrm{~mm}-2 \mathrm{~cm})$ in cecum, colon and rectum. These lesions appeared to be hardened, ulcerated, and covered by a fibrin clot. Histopathologic examination confirmed the clinical suspicion of KS. In bronchoscopy, there were nodules in the larynx and in the supraglottic region on the left side (Figure 1h), in addition to red-violet-colored mucosa in the lateral wall and medial segmentation in the middle lobe bronchus (MLB), on the right bronchial tree. The mucosa had a red-violet color at the entrance of the lingual bronchus and at the entrance of the apical segment of the upper lobar bronchus (ULB) on the left bronchial tree.

In addition, it is important to clarify that he only accepted to initiate ART, with the drugs Tenofovir (TDF), Lamivudine (3TC) and Dolutegravir (DTG), in December, after the Kaposi's exacerbation. The patient started his first chemotherapy session with Paclitaxel at the end of December 2018, and was discharged after 10 days (Figure 1i) with sulfamethoxazole-trimethoprim prophylaxis, and has been followed-up by oncologists, dermatologists and infectious diseases oncologists, dermatologists and infectious diseases specialists.

This year (2019), the patient has been clinically improving after the beginning of the treatment. More recently, control exams presented CD4+ lymphocyte count of 45 cells $/ \mathrm{mm}^{3}, \mathrm{~T}$ CD8+ 850 cells $/ \mathrm{mm}^{3}, \mathrm{CD} 4+/ \mathrm{CD} 8+$ ratio of 0.05 and viral load of $52 \mathrm{mmc}$ copies. A significant reduction in viral load at nine months with satisfactory disease evolution compared to baseline is highlighted.

\section{DISCUSSION}

The disseminated presentation of AIDS-associated KS has an extremely poor prognostic scenario in the vast majority of cases, spreading rapidly and progressively and often being fatal ${ }^{3}$. Diagnosis by histopathological examination is the gold standard ${ }^{4}$ and the early diagnosis is of essential importance for the elaboration of an efficient therapeutic strategy for the control of the disease. The disseminated form of the disease may manifest on the skin, oral mucosa, gastrointestinal tract, lymph nodes and lungs ${ }^{5}$ similar to the patient presented here. This symptomatology, CD4 cell count lower than 150 cells per mm3, the presence of systemic disease, as well as pulmonary involvement exhibiting T1, I1 and S1 staging are high risk factors and they have been associated with a poor outcome and patients' death ${ }^{6}$.

In these cases, chemotherapy associated with ART is very important for the treatment and follow-up of patients, as the percentage of $\mathrm{KS}$ in patients who do not have immunodeficiencies are relatively uncommon ${ }^{5}$. Paclitaxel is an accepted option as the treatment of choice associated with "pegylated liposomal doxorubicin", but it has a higher myelotoxic potential, may cause total alopecia and also requires care due to the possibility to increase HAART reactions ${ }^{6}$.

The differencial diagnosis of Kaposi's sarcoma mimickers includes deep fungal infections, vascular malignant neoplasms and vasculopathic disorders. Cutaneous manifestations of pneumocystosis may also show violaceous papules and nodules, but will also have pulmonar involvement and the Gomori methenamine-silver (GMS) staining will show typical cysts of Pneumocystis jirovecci $i^{7}$. In addition, Zygmycosis or Mucormycosis, an Entomophtoromycosis will also presentviolaceous nodules in diabetic patients, in extensive burn or trauma areas and GMS is the best method to evidence fungal hyphae in tissues due to a high contrast with minimal background impregnation. The histopathological examination revealed tissue alterations, such as mixed inflammatory infiltrate, necrosis, thrombosis and angioinvasion by the fungal hyphae $^{8}$. Angiosarcoma, a malignant vascular tumor, shows nuclear pleomorphism and atypical vascular channels, so it can easily be distinguished from KS by histopathology. Calciphylaxis shows occlusion of vascular vessels in subcutaneous fat with calcium deposition. In conclusion, when a patient presents with violaceous papules and nodules, the clinical investigation (appearance of lesions and the patient's history) will be best complemented by histopathological analyses.

\section{AUTHORS' CONTRIBUTIONS}

Carla Andrea Avelar Pires: revised the manuscript critically; Julius Caesar Mendes Soares Monteiro: took part in conception and design of the study; Rhyan Meninea Rego: responsible for acquisition of data; Victoria Juliana Campos Lodi: drafted the manuscript.

\section{CONFLICT OF INTERESTS}

The authors declare that they have no conflict of interest.

\section{ETHICAL APPROVAL}

All procedures performed in studies involving human participants were in accordance with the ethical standards of the Universidade Federal do Estado do Para Ethics Committee, with the 1964 Helsinki declaration and its later amendments or comparable ethical standards and finally 
with Resolution 466/2012 of the Brazilian National Health Council (CNS) on Ethics in Research with human beings.

\section{FUNDING}

The authors declare that they have received no funds.

\section{INFORMED CONSENT}

Informed consent was obtained from this participant, included in the study.

\section{REFERENCES}

1. Joint United Nations Program on HIV/AIDS. Prevention gap report. Geneva: UNAIDS; 2016. [cited 2019 Dec 4]. Available from: www.unaids.org/en/resources/documents/2016/ prevention-gap

2. Schwartz RA. Kaposi's sarcoma: an update. J Surg Oncol. 2004;87:146-51
3. Gonçalves PH, Uldrick TS, Yarchoan R. HIV-associated Kaposi sarcoma and related diseases. AIDS. 2017;31:1903-16.

4. Antman K, Chang Y. Kaposi's sarcoma. New Engl J Med. 2000;342:1027-38

5. Nasti G, Talamini R, Antinori A, Martellotta F, Jacchetti G, Chiodo F, et al. AIDS-related Kaposi's sarcoma: evaluation of potential new prognostic factors and assessment of the AIDS Clinical Trial Group Staging System in the Haart Era, the Italian Cooperative Group on AIDS and Tumors and the Italian Cohort of Patients Naive From Antiretrovirals. J Clin Oncol. 2003;21:2876-82.

6. Bhutani M, Polizzotto MN, Uldrick TS, Yarchoan R. KSHVassociated malignancies: epidemiology, pathogenesis, and advances in treatment. Semin Oncol. 2015;42:223-46.

7. Blanco JL, Garcia ME. Immune response to fungal infections. Vet Immunol Immunopathol. 2008;125:47-70.

8. Prabhu RM, Patel R. Mucormycosis and entomophthoramycosis: a review of the clinical manifestations, diagnosis and treatment. Clin Microbiol Infect. 2004;10 Suppl 1:31-47. 